\title{
Fast Multivariate Ordinal Type Histogram Matching
}

\author{
Sung-Hyuk Cha \\ Department of Computer Science, Pace University \\ 861 Bedford rd, Pleasantville, NY 10570 \\ scha@pace.edu
}

\begin{abstract}
When patterns are represented as histograms, the earth mover's distance, EMD has been considered an excellent metric between two distributions. EMD is formulated as the transportation problem which is a hard optimization problem. In similarity based pattern retrieval problems, computing EMDs for all histograms in the database against a query histogram would take too long time for users to wait for the output. Hence, the candidate selection technique is presented to speed up the EMD based multivariate ordinal type histogram retrieval problem. It guarantees to find all similar histograms while achieving significant speed up. Theoretical relationships between other metrics for multivariate histograms and their usages are presented as well.
\end{abstract}

\section{Introduction}

Distance between histograms plays important role in classification, clustering, and retrieval problems [1]. Among numerous metrics, the Earth Mover's Distance, or $E M D$ in short, has received great attention in computer vision and pattern recognition [2-4]. It is the minimal amount of work that must be performed to transform one distribution into the other by moving "distribution mass" [2]. This concept was called the Mallows distance in Statistics earlier [3]. Other conceptually similar metrics includes the edit distance [5,6] which appeared in literature simultaneously as EMD. Only univariate histograms were considered and depending on the measurement types, $\Theta(b), \Theta(b)$, and $O\left(b^{2}\right)$ efficient solutions for computing nominal, ordinal and modulo type histogram edit distances were discovered [5-7] where $b$ is the number of bins.

When histograms are multivariate, computing the cost minimization can be formulated as a transportation problem [2] which is a hard optimization problem. One of the solutions is the Simplex method [9] and the efficient algorithm takes $O\left(b^{3} \log b\right)$ [9]. Consider the similarity based histogram retrieval problem, i.e., retrieving similar histograms out of $w$ histograms in the database to a query histogram, $q$ using the $E M D$. A naïve way to solve the problem would be running the simplex method or equivalent algorithm $w$ times which takes unbearably long time for users to wait for the output.

For this reason, a fast method which quickly eliminates most histograms from consideration as possible matches is presented. Let a match be a similar histogram $h$ whose $\operatorname{EMD}(h, q) \leq t$, a threshold value. Suppose we have another distance measure, $d_{x}$ such that $d_{x}(h, q) \leq E M D(h, q)$ where $d_{x}$ can be computed very fast. Then we can use 
$d_{x}$ to eliminate $h$ from consideration if $d_{x}(h, q)>t$. This candidate selection method guarantees to find all matches while achieving significant speed up. The candidate selection technique was applied successfully for univariate ordinal [6] and modulo [10] type histograms. Here, two efficient multivariate histogram metrics whose computational complexities are linear are introduced for the multivariate ordinal type histogram retrieval problem. Theoretical relationships between metrics are presented.

The rest of the paper is organized as follows. In section 2, the edit distance between univariate histograms and the EMD between 2D cases are reviewed. Section 3 introduces two other metrics which can be computed very fast for multivariate histograms. Section 4 contains use of two efficient metrics in the similarity based histogram retrieval problem. Finally, section 5 concludes this work.

\section{Preliminary: Ordinal Type Histograms}

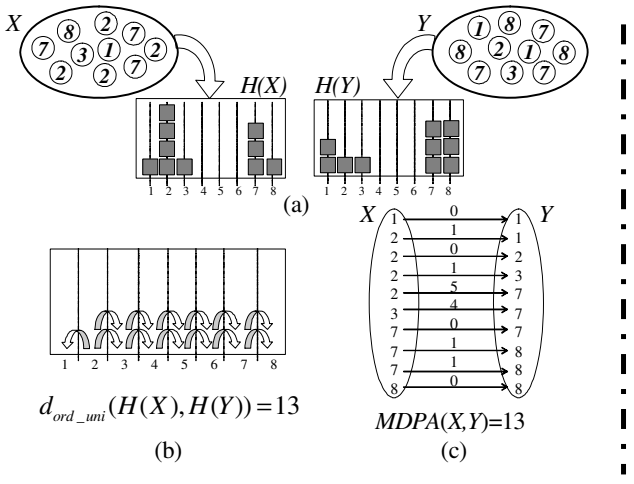

Fig. 1. Univariate ordinal type histogram and its metric

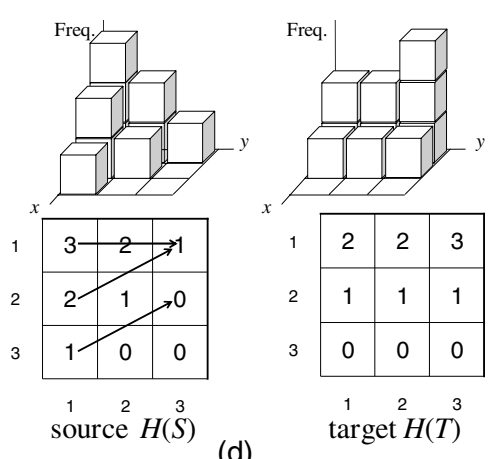

Fig. 2. 2-dimensional $3 \times 3$ ordinal histograms

\subsection{Univariate Ordinal Type Histograms}

Let $X$ be a set of $n$ elements whose possible values are finite. A histogram $H(X)$ of a set $X$ represents the frequency of each value as shown in Figure 1 (a). The frequency value of the $i$ th bin is denoted as $H_{i}(X)$, e.g., $H_{2}(X)=4$ and $H_{1}(Y)=2$ in Figure 1 (a). Let $b$ be the number of bins and if $b$ is strictly ordered and increases linearly, then the histogram is univariate ordinal type where values are simple scalar values.

Considering a histogram as a block world, the edit distance is the minimum amount of necessary movements to build one source histogram to the other target histogram by moving blocks left or right [7] as illustrated in Figure 1 (b) and the formula is:.

$$
d_{\text {ord_uni }}(H(X), H(Y))=\sum_{i=1}^{b}\left|\sum_{j=1}^{i} H_{j}(X)-H_{j}(Y)\right|
$$

The edit distance is calculated by taking the sum of absolute values of prefix sum of difference for each level. Its computational complexity is $\Theta(b)$. Proofs for the correctness and complexity of the algorithm can be found in [7 9]. Note that the edit 
distance is one of the special cases of the $E M D$ in section 2.2. It can be computed as a transportation problem [2] or the minimum difference of pair assignment problem (MDPA) [7] which can be computed in $O\left(n^{3}\right)$ using the Hungarian method [11] as depicted in Figure 1 (c). The minimum amount of necessary movements in histograms is the same as the minimum pair assignments in sets. It is important to conceive the eqn (2) because later arguments can be easily proven by the $M D P A$ concept.

$$
\operatorname{EMD}(H(X), H(Y))=\operatorname{MDPA}(X, Y)
$$

\subsection{Two Dimensional Case}

In higher dimensional histograms, values of elements are vectors rather than scalars. If each axis in the vector is ordinal and has a finite value, then the histogram is a $d$ dimensional ordinal type histogram. A 2-D ordinal type histogram has the number of bins, $b=r \times c$ and each axis is ordinal, i.e., $x=(1, \ldots, c)$ and $y=(1, \ldots, r)$.

Consider $3 \times 3$ ordinal type histograms where $n=10$ in Figure 2 as an illustrative example. One can move blocks in the source histogram $H(S)$ to make the target histogram $H(T)$ by moving one block from $(1,1)$ to $(1,3)$, one from $(2,1)$ to $(1,3)$, and one from $(3,1)$ to $(2,3)$. Let every rectangular coordinate bin in $H(S)$ be the source and that in $H(T)$ be the destination. Supply and demand values are bounded $(0 \sim n)$. Now the metric between two histograms can be formulated as a standard form of Linear Programming although it can be shortened as discussed in the later section.

$$
\begin{array}{cl}
d_{c m}(H(S), & H(T))=Z \\
\text { Minimize } & Z=\sum_{i=(1,1)}^{(r, c)} \sum_{j=(1,1)}^{(r, c)} c_{i j} x_{i j} \\
\text { s. t. } & \sum_{j=(1,1)}^{(r, c)} x_{i j}=H_{i}(S), \text { for } i=(1,1), \ldots,(r, c) \\
& \sum_{i=(1,1)}^{(r, c)} x_{i j}=H_{j}(T), \text { for } j=(1,1), \ldots,(r, c) \\
\text { and } & x_{i j} \geq 0, \quad \text { for all } i \text { and } j
\end{array}
$$

Let $d_{\text {ordeuc }}$ and $d_{\text {ord_cbd }}$ be the EMDs with the cost matrix $c_{i, j}$ using the Euclidean, $L_{2}$ and the city block $L_{1}$ distances between two grid indices, respectively. Two feasible solutions for $d_{\text {ord_euc }}(H(S), H(T))=6.48$ and one for $d_{\text {ord_cbd }}(H(S), H(T))=8$ are given in Figure 3.

Lemma 1: $d_{\text {ord_euc }}(H(X), H(Y)) \leq d_{\text {ord_cbd }}(H(X), H(Y))$.

Proof: $M D P A_{\text {ord_cbd }}(X, Y)=d_{\text {ord_cbd }}(H(X), H(Y))$. Let each pair assignment in the $M D P A_{\text {ord_cbd }}(X, Y) \quad$ setting be $a_{i}$. Then $M D P A_{\text {ord } \_c b d}(X, Y)=\sum_{i=1}^{n} L_{1}\left(a_{i}\right)$. Let $\operatorname{TEMP}(X, Y)=\sum_{i=1}^{n} L_{2}\left(a_{i}\right)$. Then $\operatorname{TEMP}(X, Y) \leq M D P A_{\text {ord_cbd }}(X, Y) \quad$ because $L_{2} \leq L_{1}$. $M D P A_{\text {ord_euc }}(X, Y) \leq T E M P(X, Y)$ by definition. $M D P A_{\text {ord_euc }}(X, Y) \leq M D P A_{\text {ord_cbd }}(X, Y)$ 


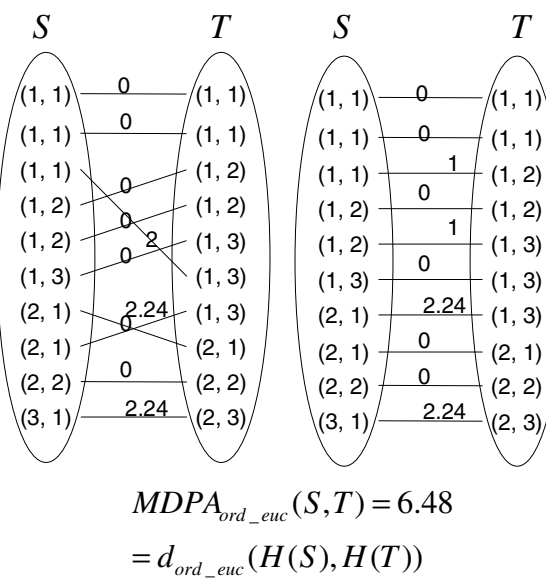

(a) (b)

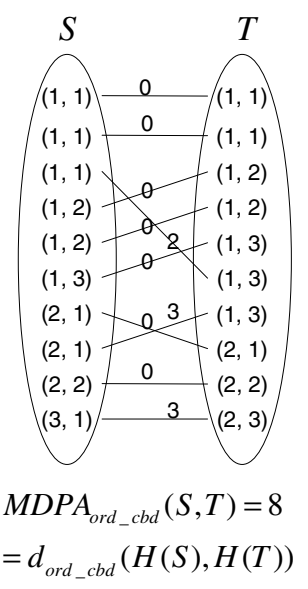

(c)

Fig. 3. (a) (b) 2 feasible solutions for $d_{\text {ord_euc }}$ (c) $d_{\text {ord_cbd }}$

\section{Efficient Metrics}

This section introduces two metrics with linear time or lower complexities which shall be used to speed up the EMD based histogram retrieval.

\subsection{Sum of Direct Subtractions (SDS)}

Consider the cost matrix such that the diagonal cells have all 0's and all others have 1's. Histograms with such a cost matrix were referred to nominal type histograms [7]. MDPA for our examples is given in Figure 4 (a). Let $d_{s d s}$, the sum of direct subtractions, $S D S$ in short, be the $E M D$ with such a cost matrix and its equation is:

$$
d_{s d s}(H(S), H(T))=n-\sum_{i=1}^{b} \min \left(H_{i}(S), H_{i}(T)\right)=\frac{\sum_{i=1}^{b}\left|H_{i}(S)-H_{i}(T)\right|}{2}
$$

The eqn (4) is often known as the non-intersection. Its computational complexity is linear: $\Theta(b)=\Theta(r \times c)$. The term, $\Sigma|H(S)-H(T)|$ is the city block distance, $L_{1}(H(S), H(T))=2 \times d_{s d s}(H(S), H(T))$ [7]. It should be noted that $L_{1}(H(S), H(T)) \neq$ $d_{\text {ord_cbd }}(H(S), H(T))$ although the term 'city block distance' appears in both metrics.

Let $D$ be the direct subtraction of $H(T)$ from $H(S) . D=H(S)-H(T)$ contains positive and negative numbers as shown in Figure 4 (b). Let $D P$ and $D N$ be the sets of elements in positive and negative positions in $D$, respectively. Every element in $D P$ must be assigned to a certain element in $D N$ as shown in Figure 4 (c). Each assignment cost is $1 . S D S$ is simply the cardinality of either $D P$ or $D N ; d_{s d s}(H(S), H(T))=|D P|=|D N|$.

Readers might easily notice that the constraints in eqn (3) can be reduced. $H(S)$ and $H(T)$ can be reduced to $H(D P)$ and $H(D N)$, respectively and non-zero cells in $H(D P)$ and $H(D N)$ are only necessary sources and destinations as shown in Figure 4 (d).

$$
\operatorname{MDPA}(D P, D N)=\operatorname{MDPA}(S, T)
$$




$$
\operatorname{EMD}(H(D P), H(D N))=E M D(H(S), H(T))
$$

$E M D$ may be computed efficiently by the constraint reduction but there is no asymptotical impact on average. It is stated because it simplifies proofs for following lemmas.

Lemma 2: $d_{\text {sds }}(H(S), H(T)) \leq d_{\text {ord_euc }}(H(S), H(T))$

Proof: Let $a \in D P$ and $b \in D N . c_{a, b}=1$ for $M D P A_{s d s}(D P, D N)$ whereas $c_{a, b} \geq 1$ for $M D P A_{\text {ord_euc }}(D P, D N)$. Hence, $M D P A_{\text {sds }}(D P, D N) \leq M D P A_{\text {ord_euc }}(D P, D N)$. Thus $M D P A_{\text {sds }}(S, T) \leq M D P A_{\text {ord_euc }}(S, T)$.

Lemma 3: $d_{s d s}(H(S), H(T)) \leq d_{\text {ord_cbd }}(H(S), H(T))$.

Proof: the similar argument to that of Lemma 2.

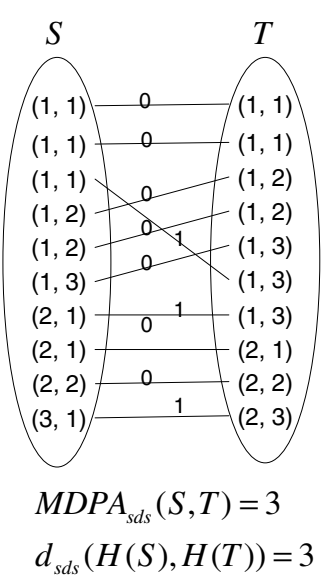

(a)

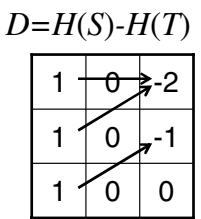

(b)

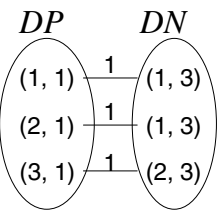

(c)

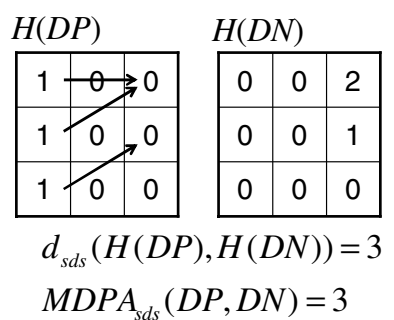

(d)

Fig. 4. $S D S$ for nominal type histograms

\subsection{Sum of Grid Differences (SGD)}

Every vertical or horizontal grid dissects the histogram into two parts as shown in Figure 5 (a). In order to edit a source histogram to a target histogram, at least eqns (7) or eqn (8) amount of movements must pass the horizontal or vertical grid.

$$
\begin{aligned}
& g d(k, *)=\left|\sum_{i=1}^{k} H_{(i, *)}(S)-\sum_{i=1}^{k} H_{(i, *)}(T)\right| \\
& g d(*, k)=\left|\sum_{i=1}^{k} H_{(*, i)}(S)-\sum_{i=1}^{k} H_{(*, i)}(T)\right|
\end{aligned}
$$

Let the sum of grid differences ( $S G D$ ) be the sum of the eqn (7) for every horizontal grid and the eqn (8) for every vertical grid.

$$
d_{s g d}(H(S), H(T))=\sum_{i=1}^{r-1} g d(i, *)+\sum_{i=1}^{c-1} g d\left({ }^{*}, i\right)
$$


It can be very efficiently computed. Let $H^{1}(X), \ldots, H^{d}(X)$ be projection histograms the histogram is $d$-dimensional histogram. Note that each $H^{i}$ is an univariate ordinal type histogram. Thus, $S G D$ can be defined using the eqn (1):

$$
d_{s g d}(H(S), H(T))=\sum_{i=1}^{d} d_{\text {ord_uni }_{-}}\left(H^{i}(S), H^{i}(T)\right)
$$

In 2D histograms,

$$
=\sum_{i=1}^{r}\left|\sum_{j=1}^{i} H_{j}^{x}(S)-H_{j}^{x}(T)\right|+\sum_{i=1}^{c}\left|\sum_{j=1}^{i} H_{j}^{y}(S)-H_{j}^{y}(T)\right|
$$

where $H^{x}$ and $H^{y}$ are vertical and horizontal projection histograms.

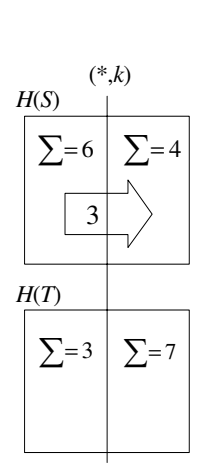

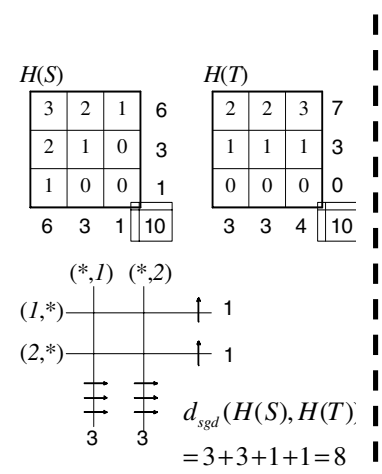

Fig. 5. Grid difference distance

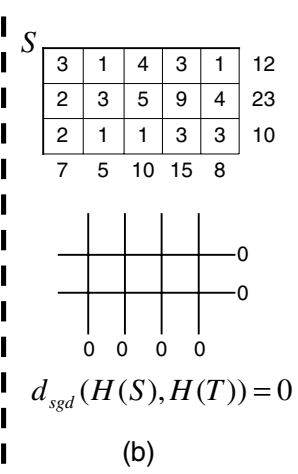

(a)
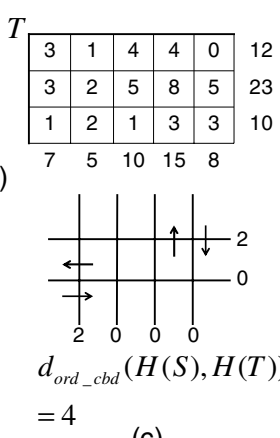

(c)

Fig. 6. Example for Lemma 4

The computational complexity is clearly $\Theta(d \times b)$ as one must build the projection histograms for each axis. When $d$ is a small constant value like 2 or $3, \Theta(b)$. Note that in many applications, the projection histograms might be pre-computed before matching. In this case, the complexity for $S G D$ is $\Theta(r+c) \approx \Theta(\sqrt{b})$ for $2 \mathrm{D}$ and $\approx \Theta(d \times \sqrt[d]{b})$ for $d$-dimensional histograms $)$.

Lemma 4: $d_{s g d}(H(S), H(T)) \leq d_{\text {ord_cbd }}(H(S), H(T))$

Proof: Let $a g(*, k)$ and $a g\left(k,{ }^{*}\right)$ be the number of blocks that actually pass the $k$ th vertical and horizontal grid, respectively when the histogram is edited using the city block distance cost matrix. Then

$$
d_{\text {ord_cbd }}(H(S), H(T))=\sum_{i=1}^{r-1} a g\left(i,{ }^{*}\right)+\sum_{i=1}^{c-1} a g(*, i)
$$

ag cannot be less than $g d$ for every grid. If so, there is no way that the edited source histogram can be the same as the target histogram. However, $a g \geq g d$ because certain blocks can move in different positions on a certain grid in the handshaking manner as shown in Figure 6 (c).

Fallacy 1: $d_{s g d}(H(S), H(T)) \leq d_{\text {ord_euc }}(H(S), H(T))$. 
Proof: a counter example is given in Figure 2, $d_{\text {sgd }}(H(S), H(T))>d_{\text {ord_euc }}(H(S), H(T))$, i.e., $8>6.48$ while there are other examples like in Figure 6, $d_{\text {sgd }}(H(S), H(T)) \leq$ $d_{\text {ord_euc }}(H(S), H(T))$, i.e., $0<4$

Although the 2D cases are used for the simplicity sake, all claims are valid for any dimensional ordinal type histograms. It should be noted that $S G D$ is only for multivariate ordinal type histograms but not for any other type histograms.

\section{Multivariate Histogram Retrieval}

Consider a query, "Find all similar histograms in a database $D$ to a given histogram $q$." This problem can be tackled by defining metrics between histograms, preferably $d_{\text {ord_euc }}$. When a threshold value $t$ is given, the problem can be formalized as:

$$
S_{\text {ord_euc }}^{t}=\forall h\left(d_{\text {ord_euc }}(q, h) \leq t \wedge h \in D\right)
$$

A naïve way to solve the problem is to compute the $d_{\text {ordeuc }}$ for every histogram $\left\{h_{1}, \ldots\right.$, $\left.h_{w}\right\}$ where $w$ is the number of histograms in $D$ which takes too long. A better way is using the candidate selection technique. Suppose we first compute the candidate set.

$$
C_{s d s}^{t}=\forall h\left(d_{s d s}(q, h) \leq t \wedge h \in D\right)
$$

Then the eqn (13) is equivalent to:

$$
S_{\text {ord_euc }}^{t}=\forall h\left(d_{\text {ord_euc }}(q, h) \leq t \wedge h \in C_{\text {sds }}^{t}\right)
$$

Instead of computing the computationally expensive $d_{\text {ord_euc }}$ for all $h$ 's in $D$, one can only compute $h$ 's in the candidate set in the eqn (15) to find matches.

In order to utilize candidate selection technique, the following necessary conditions must be met.

- $\quad C$ must be computed fast, i.e., $o\left(b^{3} \log b\right)$

- $C$ must contain all matches, i.e., $C \supset S_{\text {ord_euc }}^{t}$

The first condition is met as stated in the previous section; computing $d_{s d s}(q, \forall h)$ takes $\Theta(w b)$. Theorems of the present section are for the second necessary condition.

Theorem 1: $S_{\text {ord_euc }}^{t} \subset C_{\text {sds }}^{t}$

Proof: Consider an $h$ whose $d_{\text {ord_euc }}(q, h) \leq t$. From the Lemma $2, d_{s d s}(q, h) \leq$ $d_{\text {ord_euc }}(q, h) \leq t$. Hence, $S_{\text {ord_euc }}^{t} \subset C_{\text {sds }}^{t} \quad$ as shown in Figure 7 (a)

Suppose one is looking for all histograms in eqn (16).

$$
S_{\text {ord_cbd }}^{t}=\forall h\left(d_{\text {ord_cbd }}(q, h) \leq t \wedge h \in D\right)
$$

Then, the $S D G$.can be utilized to produce a candidate set:

$$
C_{s g d}^{t}=\forall h\left(d_{s g d}(q, h) \leq t \wedge h \in D\right)
$$

Moreover, one can also utilize the $S D S$ as another candidate set. Hence the eqn (16) is equivalent to:

$$
S_{\text {ord_cbd }}^{t}=\forall h\left(d_{\text {ord_cbd }}(q, h) \leq t \wedge h \in\left(C_{\text {sds }}^{t} \cap C_{\text {sgd }}^{t}\right)\right)
$$

Figure 7 (b) illustrates the eqn (18). 


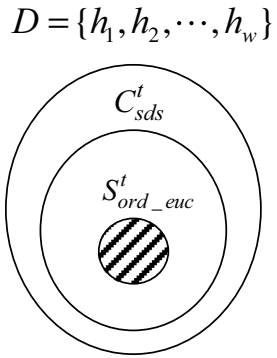

(a)

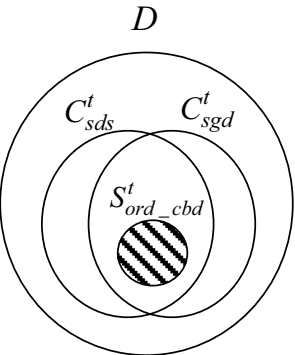

(b)

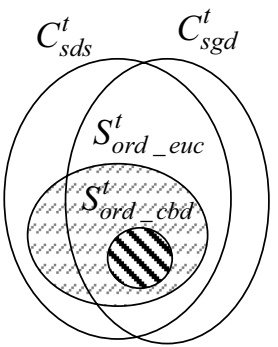

(c)

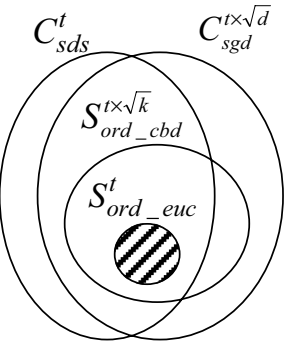

(d)

Fig. 7. Relationship between candidate sets

Theorem 2: $S_{\text {ord_cbd }}^{t} \subset\left(C_{s d s}^{t} \cap C_{s g d}^{t}\right)$

Proof: $S_{\text {ord_cbd }}^{t} \subset C_{s d s}^{t}$ by Lemma 3 and $S_{\text {ord_cbd }}^{t} \subset C_{s g d}^{t}$ by Lemma 4 .

Unfortunately as depicted in Figure 7 (c), $C_{\text {sgd }}^{t}$ cannot be a candidate set for $S_{\text {ord_euc }}^{t}$; $S_{\text {ord_euc }}^{t} \not \subset C_{\text {sgd }}^{t}$ because of Fallacy 1 . Nevertheless, there is a way to utilize the $S G D$ for $d_{\text {ordeuc }}$. Let $p$ and $q$ be points in $d$-dimensional Euclidean space and let $L_{1}$ and $L_{2}$ be the city block and Euclidean distances between two points, respectively.

Axiom 1: $L_{2}(p, q) \leq L_{1}(p, q) \leq \sqrt{d} \times L_{2}(p, q)$.

This bound can be shown by a simple geometry.

Lemma 5: $d_{\text {ord_cbd }}(H(X), H(Y)) \leq \sqrt{d} \times d_{\text {ord_euc }}(H(X), H(Y))$

Proof: Let each pair assignment in $M D P A_{\text {ord_euc }}$ be $a_{i} \cdot M D P A_{\text {ord_euc }}(X, Y)=\sum_{i=1}^{n} L_{2}\left(a_{i}\right)$. Let $\operatorname{TEMP}(X, Y)=\sum_{i=1}^{n} L_{1}\left(a_{i}\right)$, then $\sum_{i=1}^{n} L_{2}\left(a_{i}\right) \leq \sum_{i=1}^{n} L_{1}\left(a_{i}\right) \leq \sum_{i=1}^{n} \sqrt{d} L_{2}\left(a_{i}\right)$ by Axiom 1. $T E M P(X, Y) \leq \sum_{i=1}^{n} \sqrt{d} L_{2}\left(a_{i}\right)=\sqrt{d} \times \sum_{i=1}^{n} L_{2}\left(a_{i}\right)$.

$\operatorname{TEMP}(X, Y) \leq \sqrt{d} \times M D P A_{\text {ord_euc }}(X, Y)$ and $M D P A_{\text {ord_cbd }}(X, Y) \leq T E M P(X, Y)$ by definition. $\therefore \quad M D P A_{\text {ord_cbd }}(X, Y) \leq \sqrt{d} \times M D P A_{\text {ord }_{-} \text {euc }}(X, Y)$

$$
S_{\text {ord_cbd }}^{t \times \sqrt{d}}=\forall h\left(d_{\text {ord_c } c b d}(q, h) \leq t \times \sqrt{d} \wedge h \in D\right)
$$

Lemma 6: $S_{\text {ord_cbd }}^{t} \subset S_{\text {ord_euc }}^{t} \subset S_{\text {ord_c } c b d}^{t \times \sqrt{d}}$

Proof: by Lemma 1 and Lemma 5

$$
\text { Let } \quad C_{s g d}^{t \times \sqrt{d}}=\forall h\left(d_{s g d}(q, h) \leq t \times \sqrt{d} \wedge h \in D\right)
$$

Theorem 3: $S_{\text {ord_euc }}^{t} \subset\left(C_{s g d}^{t \times \sqrt{d}} \cap C_{s d s}^{t}\right)$

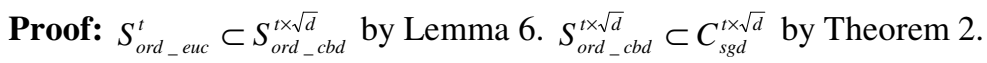


In all, computing $S_{\text {ord_euc }}^{t}$ by eqn (13) setting would take too long for users to wait for the output. Some speed up can be achieved in eqn (15) setting. Further speed up can be achieved by the following eqn (21) setting.

$$
S_{\text {ord_euc }}^{t}=\forall h\left(d_{\text {ord_euc }}(q, h) \leq t \wedge h \in\left(C_{s g d}^{t \times \sqrt{d}} \cap C_{s d s}^{t}\right)\right)
$$

Corollary 1: $\exists h\left(h \in\left(C_{s d s}^{t}-C_{s g d}^{t \times \sqrt{2}}\right)\right)$

Corollary 2: $\exists h\left(h \in\left(C_{s g d}^{t \times \sqrt{2}}-C_{s d s}^{t}\right)\right)$

Proof: Let $q, a$, and $b$ be $3 \times 3$ histograms with $n=20$.

$q=\begin{array}{rrrrrrrrr}2 & 3 & 2 & 1 & 3 & 2 & 0 & 1 & 2 \\ 1 & 2 & 3 \\ 2 & 2 & 3 & a=2 & 1 & 1 \\ 3 & 3 & 4 & b=3 & 3 & 4 \\ 1 & 4 & 2\end{array}$

If $t=4, a$ and $b$ are examples of Corollary 1 and 2, respectively. $d_{s d s}(q, a)=4 \leq t$ where $d_{s g d}(q, a)=6>t \times \sqrt{2} \cdot d_{s d s}(q, b)=6>t$ where $d_{s g d}(q, b)=5 \leq t \times \sqrt{2}$.

Corollaries 1 and 2 assure that using the eqn (21) is better than using either one of candidate sets alone. In other words, there is no need to compute $d_{\text {ord_euc }}(q, a)=$ $4.8284>t$ and $d_{\text {ord_euc }}(q, b)=6.4142>t$ because their values are guaranteed to be larger than $t$ by Theorem 3 .

For the experiments, 101 histograms were randomly generated. Using the leave one out method [1], the table 1 shows the average percentage of retrieved similar histograms. For instance of a $3 \times 5$ histogram of $n=70$ and $t=15$, only $16.79 \%$ of histograms are needed to be validated for matches. The smaller $t$, the smaller the candidate set size.

Table. 1. Average candidate and match set sizes in $\%$

\begin{tabular}{|c|c|c|c|c|c|c|}
\hline$r \times c$ & $N$ & $t$ & $\left|C_{s d s}^{t}\right|$ & $\left|C_{s g d}^{i x \sqrt{2}}\right|$ & $\left|C_{s g d}^{t \mid x \sqrt{2}} \cap C_{s d s}^{t}\right|$ & $\left|S_{\text {ord_euc }}^{t}\right|$ \\
\hline $3 \times 5$ & \begin{tabular}{c|c|}
50 \\
\end{tabular} & $\frac{10}{10}$ & $7.9 \%$ & $21.4 \%$ & $4.65 \%$ & $0.38 \%$ \\
\hline $3 \times 5$ & 50 & 12 & $23.9 \%$ & $29.7 \%$ & $13.60 \%$ & $2.75 \%$ \\
\hline $3 \times 5$ & 70 & 12 & $6.8 \%$ & $18.3 \%$ & $3.45 \%$ & $0.55 \%$ \\
\hline $3 \times 5$ & 70 & 15 & $27.3 \%$ & $41.1 \%$ & $16.79 \%$ & $3.11 \%$ \\
\hline $5 \times 7$ & 100 & 28 & $16.2 \%$ & $30.8 \%$ & $7.94 \%$ & $0.12 \%$ \\
\hline $5 \times 7$ & 100 & 30 & $30.3 \%$ & $37.6 \%$ & $15.43 \%$ & $0.30 \%$ \\
\hline
\end{tabular}

The algorithm using the candidate selection technique for the $d_{\text {ordeuc }}$ based $d$ dimensional ordinal type histogram retrieval problem is:

\begin{tabular}{|c|}
\hline Input: $q, D$, and $t$. \\
\hline Output: $S_{\text {ord_euc }}^{t}=M$. \\
\hline Procedure: \\
\hline 1. $M=\varnothing$ \\
\hline 2 for $i=1$ to $w$ \\
\hline if $d_{s g d}\left(q, h_{i}\right) \leq \sqrt{d} \times t$ \\
\hline if $d_{s d s}\left(q, h_{i}\right) \leq t$ \\
\hline if $d_{\text {ord_euc }}\left(q, h_{i}\right) \leq t$ \\
\hline$h_{i} \in M$ \\
\hline 7 return $M$ \\
\hline
\end{tabular}


But for lines 3 and 4, it would take too long. The order of these lines matters. If the projection histograms for every histogram in $D$ are pre-computed, $d_{s g d}(q, \forall h)$ takes $\Theta(w \times(r+c))$ much faster than $d_{s d s}(q, \forall h)$ which takes $\Theta(w \times(r \times c))$ for 2D histograms and thus $S G D$ should precede $S D S$. Then the running time complexity for the algorithm is $\Theta(w \times(r+c))+\Theta\left(\left|C_{s g d}^{t \times \sqrt{2}}\right| \times(r \times c)\right)+\mathrm{O}\left(\left|C_{s g d}^{t \times \sqrt{2}} \cap C_{s d s}^{t}\right| \times b^{3} \log b\right)$. With a little modification, the above algorithm can be used for the instance based classifier [1]. However, in most classification problems, the threshold value is not given. One may set $t=\min \left(d_{s g d}(q, h)\right)$ and slowly increase $t$ to find the nearest one.

\section{Conclusion}

Similarity based multivariate histogram retrieval problem using the $E M D$ as a metric was considered. The candidate selection method which quickly eliminates most histograms from consideration as possible matches was presented. All matches, i.e., similar histograms to a query histogram are still guaranteed to be found while achieving significant speed up. Main contribution of this article is introducing two metrics which satisfy the two necessary conditions to produce a candidate set $C$ for the $E M D$ based multivariate histogram retrieval problem.

In addition to aforementioned two necessary conditions for the candidate selection method, a desirable condition is that the expected $|C|$ must be small, i.e., $|C|<<|D|$. Although Theorems in this paper imply $|C| \leq|D|$, finding the expected $|C|$ theoretically which requires probability analysis is one of the open problems. Finding a new candidate selection function such that the expected $|\mathrm{I}|$ is small is also a challenging problem, e.g., an easy one might be finding a tighter bound in Lemma 5.

An example of 3D ordinal type histograms is the $R G B$ histogram because each component histogram is ordinal type. Consider $H S V$ histogram which is a multivariate heterogeneous type histogram. The Hue component histogram is modulo type whereas Saturation and value components are ordinal type. Dealing with heterogeneous type multivariate histograms is another open problem.

\section{References}

1. Duda, R.O., Hart, P.E., Stork, D.G.: Pattern Classification, 2nd edn. Wiley, New York (2000)

2. Rubner, Y., Tomasi, C., Guibas, L.J.: A metric for distributions with applications to image databases. In: Proc. of ICCV, pp. 59-66 (1998)

3. Levina, E., Bickel, P.: The Earth Mover's distance is the Mallows distance: some insights from Statistics. In: Proc. Of ICCV, pp. 251-256 (2001)

4. Puzicha, J., Rubner, Y., Tomasi, C., Buhmann, J.M.: Empirical evaluation of dissimilarity measures for color and texture. In: Proc. of ICCV, pp. 1165-1173 (1999)

5. Cha, S.-H.: Fast Image Template and Dictionary Matching Algorithms. In: Chin, R., Pong, T.-C. (eds.) ACCV 1998. LNCS, vol. 1351, pp. 370-377. Springer, Heidelberg (1997)

6. Cha, S.-H.: Efficient Algorithms for Image Template and Dictionary Matching. Journal of Mathematical Imaging and Vision 12(1), 81-90 (2000) 
7. Cha, S.-H., Srihari, S.N.: On Measuring the Distance between Histograms. Journal of Pattern Recognition 35(6), 1355-1370 (2002)

8. Hiller, F.S., Lieberman, G.J.: Introduction to mathematical programming, 2nd edn. McGraw-Hill, New York (1995)

9. Ahuha, R.K., Magnanti, T.L., Orlin, J.B.: Network Flows. Prentice-Hall, Englewood Cliffs (1993)

10. Cha, S.-H.: A fast hue-based colour image indexing algorithm. Machine GRAPHICS \& VISION 11(2/3), 285-295 (2002)

11. Kuhn, H.W.: The Hungarian method for the assignment problem. Naval Research Logistics Quarterly 2, 83-97 (1955) 